\title{
On relay placement for deterministic line networks
}

\author{
R. Appuswamy \\ UCSD \\ rathnam@ucsd.edu
}

\author{
E. Atsan \\ EPFL \\ emre.atsan@epfl.ch
}

\author{
C. Fragouli \\ EPFL \\ christina.fragouli@epfl.ch
}

\author{
M. Franceschetti \\ UCSD \\ massimo@ece.ucsd.edu
}

\begin{abstract}
We consider a unicast communication problem where, a source transmits information to a destination through a wireless network with the help of $k$ relays positioned on a line. We adopt the linear deterministic model to capture the wireless signal interactions and study the optimal placement of the relays so that the capacity from the source to the destination in the deterministic network is maximized. Analytical results are provided for a number of special cases, and the insights gained are used to provide a heuristic framework for designing large relay networks. ${ }^{1}$
\end{abstract}

\section{INTRODUCTION}

This paper starts to re-examine a basic communication systems design question: if we are given a source that would like to communicate with a geographically separated destination, how should we place a given number of relays between the source and the destination, to optimize the information rate that the source can reliably convey?

A traditional system would place the relays at equal distances in the line that separates the source from the destination (uniform placement). In wireless networks, transmissions are broadcasted and can be overheard not only by the intended receiver, but also from all other receivers that are close to it. Thus simultaneous transmissions interfere at the receivers, at different power levels. However, the traditional wireless communication architectures artificially simulate a wired network architecture by orthogonalizing the transmitted signals in time, frequency or through coding. Assuming equal transmit power at all nodes, orthogonalization implies that the dominating factor in the achievable performance is the maximum distance between any two consecutive nodes. Thus for traditional systems the uniform placement would lead to the best performance. If we assume that there is only one relay than can help a source-receiver pair, algorithms exist (see [8] and references therein) to find the optimal relay location under a restricted set of coding strategies at the relay node.

It is now well understood that interference contains useful information and provides a basis for cooperation among transceivers that can significantly increase the achievable rates [5]. Future wireless systems would need to leverage this information in order to satisfy the ever increasing demand to

\footnotetext{
${ }^{1}$ C. Fragouli and E. Atsan would like to acknowledge the support of the Swiss National Science Foundation Award No PP002-110483 and the ERC Starting Grant NOWIRE.

R. Appuswamy and M. Franceschetti would like to acknowledge the support of the NSF NETS grant CNS-0916778.
}

support higher rates, and indeed, recently there has been a significant research effort in wireless communications aimed at developing such cooperation techniques [6]. In this paper, we are interested in calculating the optimal relay placement in the case where interference is constructively used at the receivers.

To find the optimal placement that maximizes the capacity from the source to the destination, we need to compute the capacity for each potential relay placement.The problem of characterizing the capacity for a general wireless Gaussian network has long been an open problem. The linear deterministic network model captures the signal interactions in wireless networks. The work in [3] established an information theoretic min-cut max-flow theorem for deterministic networks, showing that the unicast capacity equals the minimum rank of the transfer matrices of cuts separating the source from the destination. Such deterministic models can provide guidelines and inspire strategies for Gaussian networks. Efficient algorithms to find coding strategies for linear deterministic networks are known [4].

In this paper, we leverage the deterministic model to study the optimal placement of relay nodes when we allow interference. Our motivation for the use of the deterministic modeling is similar to that in [3], namely, that the placement indicated from our study will help us build an intuition for the optimal placement in Gaussian networks. We present preliminary results from our ongoing work in this direction. Analytical results are provided for a number of special cases, and the insights gained there, along with simulation results, are used to provide a heuristic framework for relay placement in large relay networks.

The paper is organized as follows. Section II introduces our network and channel model and as well as our notation. Section III provides exact calculations for the optimal placement of a two-relay line network, and for a number of channel models. Section IV introduces our heuristic and examines how well it fits in a number of cases. Section V presents results in the special case where there is no fading. Finally, Section VI presents our conclusions and current work.

\section{Model and Problem Statement}

We consider a communication network where a source would like to transmit information to a destination with the help of a given number of relays. We assume that the source 
and the destination are sepated by $r$ units, and that relays can be placed on the $r-1$ positions of the line grid between the source and the destination. Moreover, we assume that all network nodes (the source and the relays) have the same transmit power.

Definition II.1. $A(k, r)$ relay network is a relay network with $k$ relays $u_{1}, u_{2}, \cdots, u_{k}$, a source node $u_{0}$ and a receiver node $u_{k+1}$. The distance between $u_{0}$ and $u_{k+1}$ is $r$ units.

For example, Fig. 1 shows a $(2, r)$ relay network. Each point to point link in a relay network is described by

$$
y=h x+z
$$

where $x$ is the transmitted signal, $y$ the received signal, $h$ the channel coefficient that captures path-loss and fading, and $z$ is Gaussian noise with distribution $\mathcal{N}(0,1)$. The capacity of the link between any pair of relay nodes at distance $r$ is given by

$$
\mathcal{C}=\frac{1}{2} \log \left(1+P_{r} S N R\right)
$$

where $P_{r}$ captures the effect of the channel between nodes at distance $r$ and $S N R$ denotes the signal to noise ratio in the absence of path loss and fading. We assume that transmissions are broadcasted, and may be received by multiple receivers at different signal strengths depending on the path loss parameters. Moreover, there is interference between transmissions, and each given receiver observes the superposition of the signals from different nodes.

\section{A. Deterministic modeling}

The linear deterministic models proposed in [1]-[3] take into account the signal interactions and represent the noise by a deterministic threshold. Thus if the capacity is $\mathcal{C}$, we assume we can receive $\lceil\mathcal{C}\rceil$ bits. Each node in a deterministic network transmits and receives a certain number of bits. We will generally denote transmitted and received bits using the variables $x$ and $y$, respectively. Each transmitted bit is broadcasted to all nodes that receive it. Interference is modeled through addition over the binary field; thus a received bit could be the binary xor of two or more transmitted bits. A cut between a source $u_{0}$ and destination $u_{k+1}$ is a partition of the nodes into sets $C$ and $\bar{C}$ with $u_{0} \in C$ and $u_{k+1} \in \bar{C}$. The channel between two nodes $u_{i}$ and $u_{j}$ is modeled as a linear transformation $\mathcal{T}_{i, j}$ which is an $n \times n$ matrix of the form

$$
\left(\begin{array}{cc}
0 & I_{\left\lceil\mathcal{C}_{i, j}\right\rceil} \\
0 & 0
\end{array}\right)
$$

where, $\mathcal{C}_{i, j}$ denotes the capacity of the channel between nodes $u_{i}$ and $u_{j}$ and is given by (1), $I_{\left\lceil\mathcal{C}_{i, j}\right\rceil}$ denotes the identity matrix of size $\left\lceil\mathcal{C}_{i, j}\right\rceil$, and 0 denotes a zero matrix of appropriate dimension. If $x_{u_{i}}$ denotes the binary vector of inputs at node $u_{i}$ and $y_{u_{j}}$ denotes the binary vector of outputs received at node $u_{j}$, then we have the following relation:

$$
y_{u_{j}}=x_{u_{i}} \mathcal{T}_{i, j}
$$

Let $A$ and $B$ be two sets of nodes. We denote by $\mathcal{T}_{A, B}$, the transfer matrix between the set of nodes $A$ and $B\left(\mathcal{T}_{A, B}\right.$ is unique up to permutation of columns and rows).

Example II.2. If $A=\left\{u_{i}\right\}$ and $B=\left\{u_{i+1}, u_{i+2}, \cdots, u_{k+1}\right\}$, then

$$
\begin{aligned}
& \mathcal{T}_{A, B}=\left(\begin{array}{llll}
\mathcal{T}_{i, i+1} & \mathcal{T}_{i, i+2} & \cdots & \mathcal{T}_{i, k+1}
\end{array}\right) . \\
& \text { Similarly, if } A=\left\{u_{i-1}, u_{i}\right\} \text { and } B= \\
& \left\{u_{i+1}, u_{i+2}, \cdots, u_{k+1}\right\} \text {, then } \\
& \mathcal{T}_{A, B}=\left(\begin{array}{cccc}
\mathcal{T}_{i, i+1} & \mathcal{T}_{i, i+2} & \cdots & \mathcal{T}_{i, k+1} \\
\mathcal{T}_{i-1, i+1} & \mathcal{T}_{i-1, i+2} & \ldots & \mathcal{T}_{i-1, k+1}
\end{array}\right) .
\end{aligned}
$$

Let $\Lambda(\mathcal{N})$ denote the set of cuts in network $\mathcal{N}$ and let $\Lambda_{l}(\mathcal{N})$ denote the following collection of layered cuts:

$$
\Lambda_{l}(\mathcal{N})=\left\{\left\{u_{0}, u_{1}, u_{2}, \ldots, u_{i}\right\}: i=1,2, \ldots, k\right\} .
$$

Definition II.3. For a binary matrix $\mathcal{T}$, let $|\mathcal{T}|$ denote its rank over $\mathbb{F}_{2}$. For a cut $C$, we define $\lambda(C)$ to be equal to $\left|\mathcal{T}_{C, \bar{C}}\right|$.

Definition II.4. For any relay network $\mathcal{N}$, define

$$
\lambda(\mathcal{N})=\min _{C \in \Lambda(\mathcal{N})} \lambda(C)
$$

and

$$
\lambda_{l}(\mathcal{N})=\min _{C \in \Lambda_{l}(\mathcal{N})} \lambda(C)
$$

The capacity of a deterministic network $\mathcal{N}$ has been shown in [1]-[3] to be equal to $\lambda(\mathcal{N})$.

\section{B. Channel Model}

We briefly describe the fading and path loss models used in this paper. We model fading using a discrete random variable $X$ which shifts the number of the received bits at a node. Let $X$ take $m$ discrete values of the form $\left\{2^{i}\right\}$ for some appropriate values of $i$ according to a discrete probability distribution $\mathcal{P}_{X}$. We assume that the communication is timeslotted and that each node is equipped with unlimited memory and can use arbitrarily long block codes. The number of bitpipes between nodes $u_{i}$ and $u_{j}$ at distance $r$ in time slot $t$ is a random variable and is given by

$$
\approx \frac{1}{2} \log \left(X_{u_{i}, u_{j}} P_{r} S N R\right)
$$

where, $P_{r}$ accounts for the path loss between $u_{i}$ and $u_{j}$ and $X_{u_{i}, u_{j}}$ is a copy of the random variable $X$ accounting for random fading.

We further assume that the signal to noise ratio is of the form $S N R=2^{2 n}$ for some positive integer $n$. We consider two different models for $P_{r}$ :

1) Exponential path loss model: $P_{r}=2^{-2 c r}$ for some positive integer $c$.

2) Power law model: $P_{r}=\frac{1}{r^{c}}$ for some positive value $c$. Furthermore, in the absence of fading, $X$ is a degenerate random variable assuming a value of 1 with probability 1 .

Since the deterministic network varies over time with each instance of the random variables $X_{u_{i}, u_{j}}$, the value of a cut $C$ in a network $\mathcal{N}$ is also a random variable. It is now easy to 


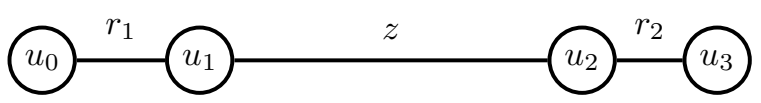

Fig. 1. Placement for two relays, where $r=r_{1}+z+r_{2}$.

show that the capacity of a grid network $\mathcal{N}$ under this model is given by

$$
\min _{C \in \Lambda(\mathcal{N})} \mathbb{E} \lambda(C) .
$$

Almost throughout the paper, analytical results are derived using the exponential path loss model which is more tractable as well as rooted in the physics of wave propagation in wireless media [7].

\section{THE TWO RELAY LINE NETWORK}

A two-relay network $\mathcal{N}_{2}$ is depicted in Fig. 1. This is the simplest network where a non-uniform placement can outperform the uniform placement ${ }^{2}$, and thus is interesting to examine in detail. The placement in this network can be described with parameters $r_{1}, z$ and $r_{2}$, with $r_{1}+z+r_{2}=r$ as depicted in Fig. 1. Fig. 2 shows the channel connections in this network. There are exactly four cuts in this network, $C_{1}=\left\{u_{0}\right\}, C_{2}=\left\{u_{0}, u_{1}, u_{2}\right\}, C_{3}=\left\{u_{0}, u_{2}\right\}$ and $C_{4}=$ $\left\{u_{0}, u_{1}\right\}$. Thus the capacity of this network equals

$$
\lambda\left(\mathcal{N}_{2}\right)=\min _{i} \mathbb{E} \lambda\left(C_{i}\right)
$$

where, the averaging accounts for fading as discussed in Section II.

We analytically calculate the optimal placement for this network for some specific channel models, and for a larger set of channels through simulation results. In all these cases we observe the same trend: when the common transmit power is low, uniform is the optimal placement. As the transmit power increases, the optimal placement has the two relays approach the source and the destination respectively. This is because the dominating cut values come from the cuts $C_{1}$ and $C_{2}$, and thus the optimal placement attempts to maximize the value for these cuts.

\section{A. Two-state fading with exponential path-loss}

Let $d$ be an integer and let $X$ be a discrete random variable that takes the values $\left\{1,1 / 2^{d}\right\}$ with equal probability. That is, we have a two-state channel model. We will distinguish between two cases, when we have small scale fading $(d \leq c)$, and when we do not $(d>c)$.

1) Small scale fading $(d \leq c)$ :

\footnotetext{
${ }^{2}$ Indeed, it is easy to see that, if $k \leq 1$, then assuming all nodes have the same transmit power and undergo independent, a uniform placement is always optimal.
}

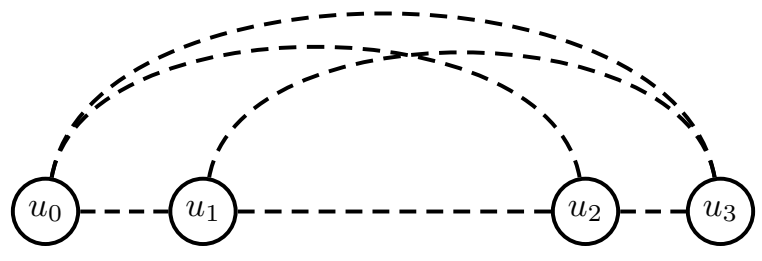

Fig. 2. Channel connections for the two relay network.

Theorem III.1. Let $\mathcal{N}$ be a $(2, r)$ line network under the small scale fading model. If $n \geq 1.6 \mathrm{cr}$, then

$$
\lambda\left(\mathcal{N}_{2}\right)=n-\frac{d}{2}-c \max \left\{r_{1}, r_{2}\right\} .
$$

Proof: We analytically calculate the cut values. For $C_{1}=$ $\left\{u_{0}\right\}$, we have

$$
\begin{aligned}
\mathbb{E} \lambda\left(C_{1}\right) & =\mathbb{E} \max \left\{n-c r_{1}-\log X_{u_{0}, u_{1}}, n-c\left(z+r_{1}\right)\right. \\
& \left.-\log X_{u_{0}, u_{2}}, n-c\left(z+r_{1}+r_{2}\right)-\log X_{u_{0}, u_{3}}\right\} \\
& =\frac{1}{2}\left(n-c r_{1}\right)+\frac{1}{2}\left(n-c r_{1}-d\right) \\
& =n-c r_{1}-d / 2
\end{aligned}
$$

Similarly, for $C_{2}=\left\{u_{0}, u_{1}, u_{2}\right\}$ and $C_{3}=\left\{u_{0}, u_{2}\right\}$, we have

$$
\begin{aligned}
& \lambda\left(C_{2}\right)=n-c r_{2}-d / 2 \\
& \lambda\left(C_{3}\right) \geq \max \left\{n-c r_{1}-d / 2, n-c r_{2}-d / 2\right\} .
\end{aligned}
$$

Finally, we consider $C_{4}=\left\{u_{0}, u_{1}\right\}$. For convenience, let $b_{i, j}=-\log X_{u_{i}, u_{j}}$ (i.e., $b_{i, j}$ is a $0 / d$-valued random variable). Let $r=z+r_{1}+r_{2}$. For all $n>c r+d$, we have

$$
\begin{aligned}
\lambda\left(C_{4}\right)=\mathbb{E} \operatorname{rk}\left(\mathcal{T}_{C_{4}, \bar{C}_{4}}\right) & \left.\left.\mathbb{E} \mathrm{rk}\left(\begin{array}{cc}
0 & I_{n-c z-b_{1,2}} \\
0 & 0
\end{array}\right] \quad\left[\begin{array}{cc}
0 & I_{n-c\left(z+r_{2}\right)-b_{1,3}} \\
0 & I_{n-c\left(z+r_{1}\right)-b_{0,2}} \\
0 & 0
\end{array}\right]\right) \quad\left[\begin{array}{cc}
0 & I_{n-c r-b_{0,3}} \\
0 & 0
\end{array}\right]\right) \\
= & \frac{3}{16}(n-c z)+\frac{3}{16}(n-c z-d) \\
& +\frac{3}{16}\left(n-c z+n-c\left(z+r_{1}+r_{2}\right)\right) \\
& +\frac{2}{16}\left(n-c z+n-c\left(z+r_{1}+r_{2}\right)-d\right) \\
& +\frac{2}{16}\left(n-c z-d+n-c\left(z+r_{1}+r_{2}\right)\right) \\
& +\frac{3}{16}\left(n-c z-d+n-c\left(z+r_{1}+r_{2}\right)-d\right) \\
= & (n-c z-d / 2)+\frac{10}{16}\left(n-c\left(z+r_{1}+r_{2}\right)-d / 2\right) .
\end{aligned}
$$

Comparing (5)-(7) with (8), for all

$$
n \geq c\left(z+r_{1}+r_{2}\right)+\frac{d}{2}+\frac{16}{10}\left(c z-c \min \left\{r_{1}, r_{2}\right\}\right)
$$

we have $\lambda\left(C_{4}\right) \geq \lambda\left(C_{i}\right)$ for $i=1,2,3$ and it follows that

$$
\lambda\left(\mathcal{N}_{2}\right)=n-\frac{d}{2}-c \max \left\{r_{1}, r_{2}\right\} .
$$



(10):

The proof of the following lemma now is immediate from

Lemma III.2. Let $\mathcal{N}$ be any $(2, r)$ line network. If $d \leq c$, then for all $n \geq 1.6 \mathrm{cr}$, the capacity of $\mathcal{N}$ is maximized by choosing $r_{1}=r_{2}=1$ and the maximum capacity is

$$
n-c-d / 2 \text {. }
$$

Now we compare the optimal placement for a $(2, r)$ line network with uniform placement of relays. When the two relays are uniformly placed between the source and the receiver,

$$
\max \left\{r_{u_{0}, u_{1}}, r_{u_{2}, u_{3}}\right\}=\left\lceil\frac{r}{3}\right\rceil \text {. }
$$

It follows that, if $d \leq c$, for all $n>c\left\lceil\frac{r}{3}\right\rceil+d / 2$, the capacity of a network with uniform relay placement is

$$
n-c\left\lceil\frac{r}{3}\right\rceil-d / 2 \text {. }
$$

Comparing (11) with Lemma III.2, the difference between optimal placement and uniform placement is (for all $n>1.6 \mathrm{cr}$ )

$$
c\left(\left\lceil\frac{r}{3}\right\rceil-1\right)
$$

which is linear in $r$.

Low SNR regime: We examine the case when $n$ is is small. From (5)-(8), we have

$$
\lambda\left(\mathcal{N}_{2}\right)=\min \left\{\lambda\left(C_{4}\right), \lambda\left(C_{1}\right), \lambda\left(C_{2}\right)\right\}
$$

If

$$
n<c\left(z+r_{1}+r_{2}\right)+d / 2+\frac{16}{10} c(z-1)
$$

then (9) is not satisfied for every choice of $r_{1}$ and $r_{2}$. In this case, a close examination of (12) reveals that $\lambda\left(\mathcal{N}_{2}\right)$ is maximized when

$$
\lambda\left(C_{4}\right)=\lambda\left(C_{1}\right)=\lambda\left(C_{2}\right) .
$$

If the node positions are constrained to be among the grid vertices, then there may not exist a choice of $r_{1}$ and $r_{2}$ that achieves (14). For analytical purposes, we solve for $r_{1}$ and $r_{2}$ after relaxing this constraint. Let $r=z+r_{1}+r_{2}$. Solving for $r_{1}$ and $r_{2}$ that achieve (14), we have

$$
\begin{aligned}
& r_{1}=r_{2} \quad\left[\text { from } \lambda\left(C_{1}\right)=\lambda\left(C_{2}\right)\right] \\
& r_{1}=r\left(\frac{1}{3}+\frac{10}{48}\right)+\frac{5}{48 c} d-\frac{10}{48 c} n . \\
& {\left[\text { from } \lambda\left(C_{4}\right)=\lambda\left(C_{1}\right) \text { and } r_{2}=r_{1}\right]}
\end{aligned}
$$

From (15), $r_{1}$ is approximately equal to $r / 3$ for small values of $n$. Thus, (15) confirms our intuition that, in the low SNR regime, placing the relays equally apart is optimal. However, it is important to notice that the optimal location for relays moves away from the center linearly with $n$. Furthermore, since $\lambda\left(\mathcal{N}_{2}\right)=\lambda\left(C_{1}\right)$, we also have

$$
\lambda\left(\mathcal{N}_{2}\right) \approx n-d / 2-\operatorname{cr}\left(\frac{1}{3}+\frac{10}{48}\right)-\frac{5}{48} d+\frac{10}{48} n
$$

where, the $\approx$ sign indicates that the above result holds only approximately since the optimal value for $r_{1}$ obtained in (15) may not be an integer.

High SNR regime: Notice that if the minimum distance between nodes is 1 (which is true for grid networks), then the capacity of a $(k, r)$ network is at most $n-c$. If $k=0$, the capacity is $n-c r-\frac{d}{2}$. If $k=1$, the capacity is $n-c \frac{r}{2}-\frac{d}{2}$. When $k=2$, we get a capacity of $n-c-\frac{d}{2}$ from (10), and thus, we have that for the two-state channel model, 2 relay nodes are almost sufficient to achieve the maximum possible capacity (which is $n-c$ ) for all $n \geq 1.6 \mathrm{cr}$. That is, by having more than 2 relays, we gain at most $d / 2$ bits whereas by increasing the number of relays from 1 to 2 , we gain $\approx \mathrm{cr} / 2$ bits.

The following theorem shows that this observation also holds in the case of square grid networks: In this case as well, adding an arbitrary number of relays, does not significantly increase the capacity as compared to adding two relays on a line networks. The proof of this theorem is provided in Appendix A.

Theorem III.3. Let $\mathcal{N}$ be a $(k, r)$ grid network. For all $n \geq$ $1.6 \mathrm{cr}$, if the relay nodes are placed optimally, then we have

$$
\begin{aligned}
n-c-d / 2 \leq \lambda(\mathcal{N}) & \leq n-c-d / 16 . & & \text { if } k \geq 2 \\
\lambda(\mathcal{N}) & =n-c\left\lceil\frac{r}{2}\right\rceil-d / 2 . & & \text { if } k=1
\end{aligned}
$$

2) Large scale fading $(d=c r)$ : We will now explicitly calculate the capacity in the case of large scale fading. Since fading is always "destructive" in our model, not surprisingly, the capacity of the two-relay network is smaller for the case of large scale fading as compared to the case when $d \leq c$. However, the optimal placement remains the same for both the fading models as $n$ increases.

The proof of this theorem is provided in Appendix B.

Theorem III.4. Let $\mathcal{N}$ be a $(2, r)$ line network under the large scale fading model, for all $n \geq 2 c r, \lambda\left(\mathcal{N}_{2}\right) \leq n-$ $\frac{1}{2} c r-\frac{3}{16} c\left(r_{1}+r_{2}\right)$.

Lemma III.5. Let $\mathcal{N}$ be any $(2, r)$ line network. If $d=c r$, then for all $n \geq 2 c r$, the capacity of $\mathcal{N}$ is maximized by choosing $r_{1}=r_{2}=1$ and the maximum capacity is

$$
n-c\left(\frac{r}{2}+\frac{3}{8}\right) \text {. }
$$

Proof: Note that the upper bound in (29) is achievable by choosing $r_{1}=r_{2}$ and it is maximized by minimizing $r_{1}+r_{2}$. Since $r_{1}, r_{2} \geq 1$, the lemma is proved.

\section{B. Simulation results}

In this section, we examine, through simulation, a more extensive family of fading distributions for the exponential as well as the power path loss model. We denote by:

- uniform 2-state the case where the fading variable $X$ takes the values $\left\{2^{-1}, 2\right\}$ with equal probability, that is, fading may affect the number of received bits by \pm 1 ,

- uniform 3-state the case where the fading variable $X$ takes the values $\left\{2^{-1}, 2^{0}, 2\right\}$ with equal probability, 
- uniform 6-state the case where the fading variable $X$ takes the values $\left\{2^{-3}, 2^{-2}, 2^{-1}, 2^{1}, 2^{2}, 2^{3}\right\}$ with equal probability, and

- Std. Normal 5-state the case where the fading variable $X$ takes the values $\left\{2^{-2}, 2^{-1}, 2^{0}, 2^{1}, 2^{2}\right\}$. We create this variable by quantizing a continuous variable $Y$ that follows the Gaussian distribution $\mathcal{N}(0,1)$ to an element of $\{-2,-1,0,1,2\}$, and define $X=2^{[Y]}$ where, $[Y]$ denotes the quantized value of $Y$.

In Fig. 3, we combine these fading distributions with the exponential path-loss model, for the two relay network. We examine the behavior of the min-cut capacity $\lambda(\mathcal{N})$ and the optimal placement as the transmit power (captured by the value $n$ ) increases.

We describe the optimal placement by providing the value for the distance $r_{1}$ between the source and the first relay (see Fig. 1). We found that the optimal placement results in $r_{1}=r_{2}$, which is a direct result of the symmetry of the tworelay configuration. The results we present are for $r=48$; thus, for low values of $n$, the optimal placement has the two relays in equal distances $\left(r_{1}=r_{2} \approx 16\right)$, while as $n$ increases the optimal placement has the two relays approach the source and the destination respectively $\left(r_{1}=r_{2} \approx 1\right)$. This behavior is consistent across all fadings, and with the analytical results. We also note that there might be several placements that result in the same optimal value for the min-cut capacity; in Fig. 3(a) we have reported the average $r_{1}$ values across all optimal placements.

Fig. 3(b) shows that the capacity increases linearly with $n$. We note that the capacity is slightly higher for the more volatile environments, i.e., when the variation of the fading is higher. We believe that this might be an artifact of the deterministic modeling: as we will see in Section V, when there is no fading, the deterministic modeling results to an alignment of the received signals at the different relays; the more variable the fading, the higher the probability we avoid this alignment. For the power law path-loss model, where the path loss effect is much smaller than in the exponential case, the optimal relay placement value $\left(r_{1}\right)$ and the capacity of the network $(\lambda(\mathcal{N}))$ are shown in Fig. 4 . We observe in this case as well a consistent behavior. The step-like behavior we see in the relay placement is a result of the nonlinear floor operation.

\section{A FRAMEWORK FOR RELAY PLACEMENT IN LARGE LINE NETWORKS}

We now discuss possible (heuristic) approaches and ideas for optimal placement of relays in large line networks. These ideas rely on the following two observations:

1) If $k \leq 2$, then our analysis in Section III show that the capacity of a $(k, r)$ line network $\mathcal{N}$ is equal to $\lambda_{l}(\mathcal{N})$. Recall from Definition II.4 that $\lambda_{l}(\mathcal{N})$ depends only on $k+1$ layered cuts. Indeed, as we will show in Section V, in the absence of fading, for an arbitrary $(k, r)$ line network (i.e., $k$ need not be 2 or less) $\mathcal{N}$, $\lambda(\mathcal{N})=\lambda_{l}(\mathcal{N})$. Simulation results seem to indicate that this property continues to hold for large line networks

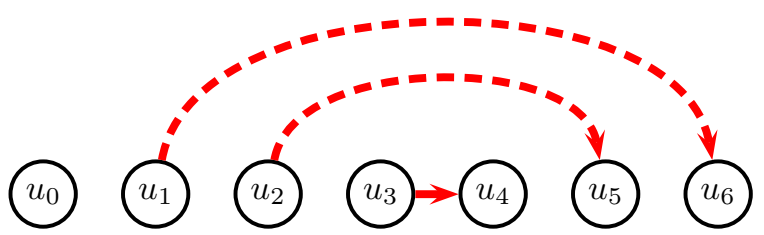

Fig. 5. For the cut $C=\left\{u_{0}, u_{1}, u_{2}, u_{3}\right\}$ in a 5 relay network, the heuristic calculation for the min-cut value sums the contribution of the channels depicted in dashed lines and ignores the remaining channels.

even in the presence of fading. This observations leads us to the following conjecture:

Conjecture IV.1. If $\mathcal{N}$ is any $(k, r)$ line network, then $\lambda(\mathcal{N})=\lambda_{l}(\mathcal{N})$.

2) Let $\mathcal{N}$ be a $(2, r)$ line network and let $C_{4}=\left\{u_{0}, u_{1}\right\}$. Again from Section III, we have

$$
\mathcal{T}_{C_{4}, \bar{C}_{4}}=\left(\begin{array}{ll}
{\left[\begin{array}{cc}
0 & I_{s_{1}} \\
0 & 0
\end{array}\right]} & {\left[\begin{array}{cc}
0 & I_{s_{2}} \\
0 & 0
\end{array}\right]} \\
{\left[\begin{array}{cc}
0 & I_{s_{3}} \\
0 & 0
\end{array}\right]} & {\left[\begin{array}{cc}
0 & I_{s_{4}} \\
0 & 0
\end{array}\right]}
\end{array}\right)
$$

for some integer random variables $s_{1}, s_{2}, s_{3}$, and $s_{4}$. We can explicitly calculate the binary rank of this matrix as follows. Let $\delta$ denote the discrete unit impulse function (i.e., $\delta(0)=1$ and $\delta(k)=0, \forall k \neq 0$ ).

$$
\begin{aligned}
& \operatorname{rk}\left(\mathcal{T}_{C_{4}, \bar{C}_{4}}\right)=s_{1}+\left(s_{2}-s_{1}\right)^{+} \\
& \quad+\left[\left(s_{3}-\left(s_{1}-s_{2}\right)^{+}\right)^{+}+\left(s_{4}-\left(s_{3}-\left(s_{1}-s_{2}\right)^{+}\right)^{+}\right.\right. \\
& \left.\left.\quad-\left(s_{2}-s_{1}\right)^{+}\right)^{+}\right]\left(1-\delta\left(\left(s_{1}-s_{3}\right)-\left(s_{2}-s_{4}\right)\right)\right) \\
& \quad+\left[s_{3}+\left(s_{4}-s_{3}\right)^{+}-\left(s_{1}+\left(s_{2}-s_{1}\right)^{+}\right)\right]^{+} \\
& \quad \times \delta\left(\left(s_{1}-s_{3}\right)-\left(s_{2}-s_{4}\right)\right)
\end{aligned}
$$

where,

$$
x^{+}= \begin{cases}x & \text { if } x \geq 0 \\ 0 & \text { if } x<0 .\end{cases}
$$

If $s_{1}$ is the largest value (which is always the case for line networks), the above expression reduces to the following: $\operatorname{rk}\left(\mathcal{T}_{C_{4}, \bar{C}_{4}}\right)$

$$
\begin{aligned}
= & s_{1}+\left[\left(s_{3}-\left(s_{1}-s_{2}\right)\right)^{+}+\left(s_{4}-\left(s_{3}-\left(s_{1}-s_{2}\right)\right)^{+}\right)^{+}\right] \\
& \times\left(1-\delta\left(\left(s_{1}-s_{3}\right)-\left(s_{2}-s_{4}\right)\right)\right) .
\end{aligned}
$$

It turns out that $\mathbb{E} \operatorname{rk}\left(\mathcal{T}_{C_{4}, \bar{C}_{4}}\right) \approx \mathbb{E}\left(s_{1}+s_{4}\right)$, i.e., the overall expected rank is well approximated by the sum of expected ranks of diagonal blocks. This observation continues to hold true for other cuts in $\mathcal{N}$ as well.

In view of the above observations, for a layered cut $C_{i}=$ $\left\{u_{0}, u_{1}, \cdots, u_{i}\right\}$ of a $(k, r)$ line network $\mathcal{N}$, we approximate 


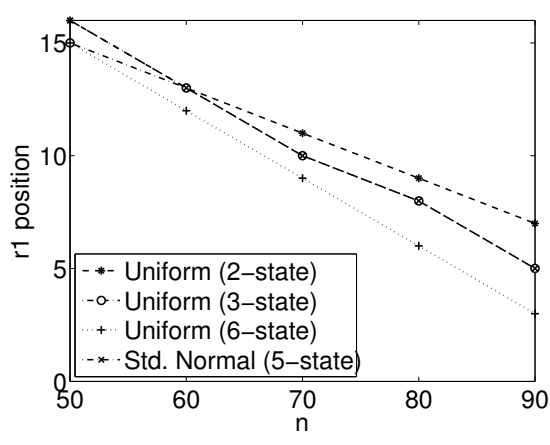

(a) Optimal relay placement

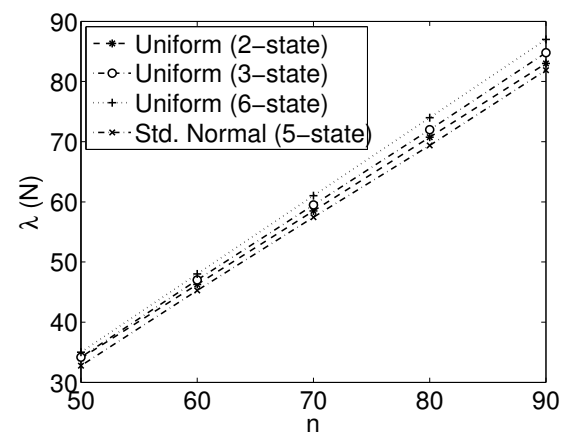

(b) Capacity $\lambda(\mathcal{N})$

Fig. 3. Simulation results for the exponential path-loss model (a) for the optimal relay 1 placement $r_{1}$, and (b) for the min-cut capacity.

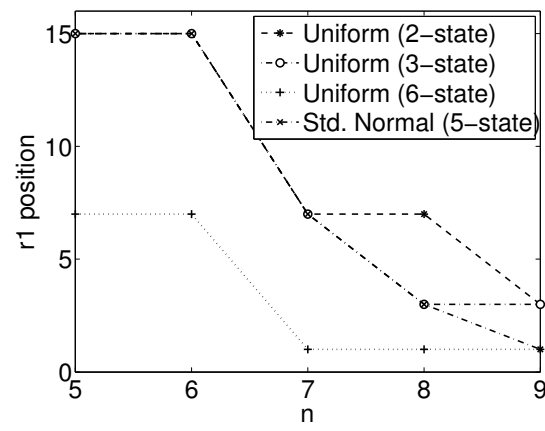

(a) Optimal relay placement

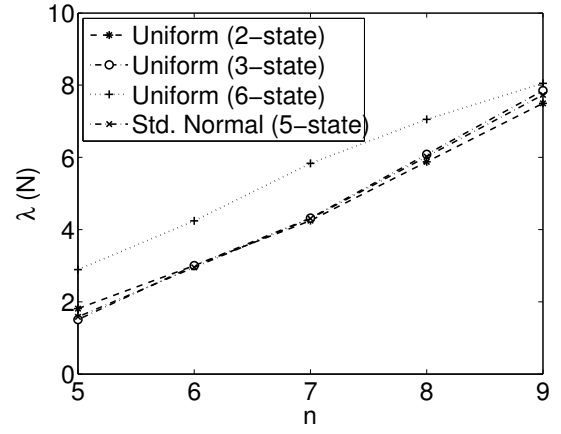

(b) Capacity $\lambda(\mathcal{N})$

Fig. 4. Simulation results for the power path-loss model for (a) for optimal relay 1 placement $r_{1}$, and (b) for the min-cut capacity.

$\mathbb{E} \lambda\left(C_{i}\right)$ by

$$
\begin{aligned}
& \sum_{j=0}^{\min \{i, k-i\}} \mathbb{E}\left|\mathcal{T}_{i-j, i+j+1}\right|= \\
& \sum_{j=0}^{\min \{i, k-i\}}\left(n-c r_{i-j, i+j+1}-\mathbb{E} \log X_{i-j, i+j+1}\right)^{+} .
\end{aligned}
$$

Graphically, we approximate $\lambda\left(C_{i}\right)$ by taking into account only the contribution of a subset of the channels that cross the cut; these are the channels that correspond to the diagonal blocks of the block transfer matrix that describes the cut. as depicted in Fig. 5 for an example network.

Let $\bar{X}=\mathbb{E} \log X_{i-j, i+j+1}$. If Conjecture IV.1 holds, we have

$$
\begin{aligned}
\lambda(\mathcal{N}) & =\min _{0 \leq i \leq k} \mathbb{E} \lambda\left(C_{i}\right) \\
& =\min _{0 \leq i \leq k} \sum_{j=0}^{\min \{i, k-i\}}\left(n-c r_{i-j, i+j+1}-\bar{X}\right)^{+} .
\end{aligned}
$$

In view of (17), the optimal relay placement problem reduces to the following optimization problem:

$$
\max _{r_{i, j}} \min _{0 \leq i \leq k} \sum_{j=0}^{\min \{i, k-i\}}\left(n-c r_{i-j, i+j+1}-\bar{X}\right)^{+} .
$$

In Fig. IV we explore how well our proposed heuristic calculation agrees with exact simulation results. We see that as the variability of the fading increases, the heuristic approximates better and better the min-cut capacity as well as the optimal node placement. This can be explained from the fact that the heuristic assumes we accummulate diversity from multiple receiving relays; i.e., it ignores the alignment that can occur for no fading (see Section V) and low variability fading.

\section{THE NO-FADING CASE}

We explore the case when there is no fading. For the exponential path loss model, and placement on the line network, we show analytically that the uniform placement is optimal. We believe that this is an artifact of the deterministic model, that allows for perfect alignment of observed signals at different relays. We have sees a similar behavior through simulations for the power path loss model. We then show that if the two relays are not placed on a line, this degenerate behavior disappears, and we can have significant benefits from a nonuniform placement.

\section{A. Exponential path-loss with no fading}

Recall that in the absense of fading, the number of links between nodes at distance $r$ is given by $\lceil n-c r\rceil$. Let $S$ denote the following $n \times n$ matrix:

$$
\left(\begin{array}{cc}
0 & I_{n-1} \\
0 & 0
\end{array}\right)
$$




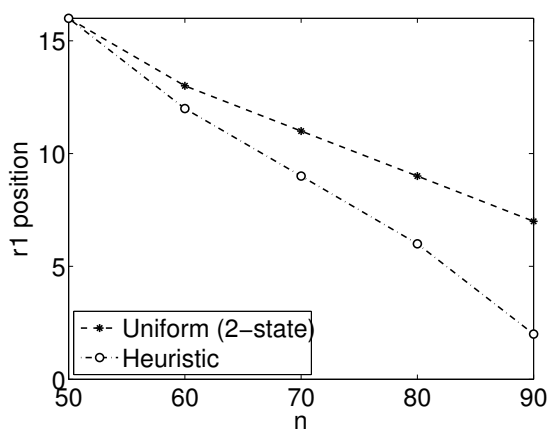

(a) r1-Uniform (2-State)

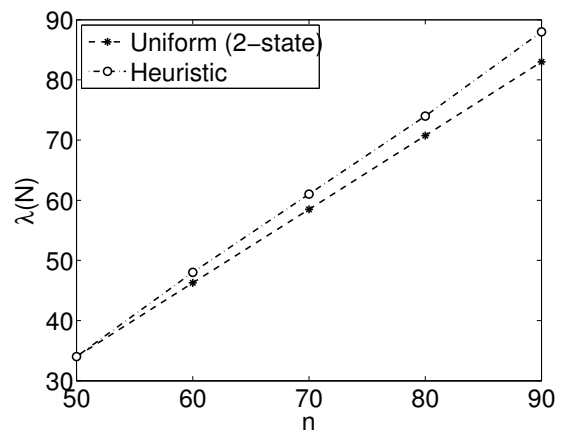

(d) capacity-Uniform (2-State)

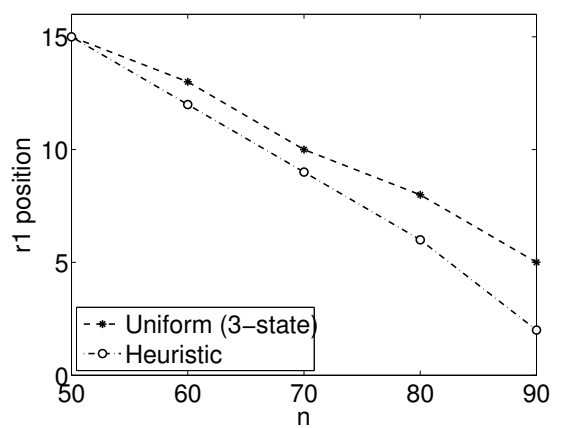

(b) r1-Uniform (3-State)

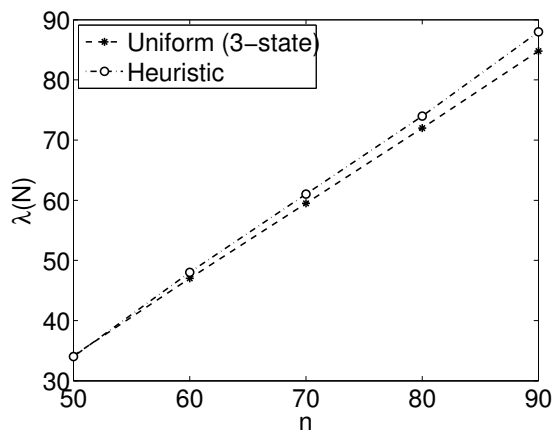

(e) capacity-Uniform (3-State)

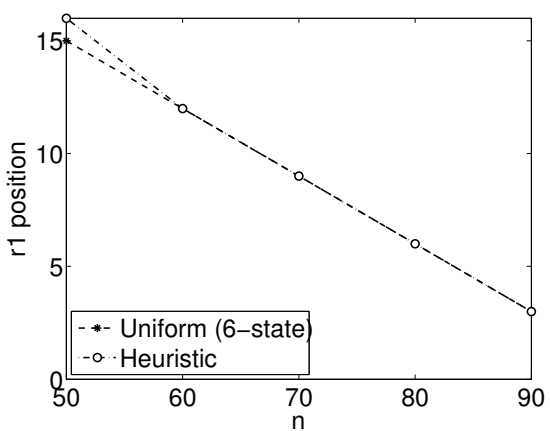

(c) r1-Uniform (6-State)

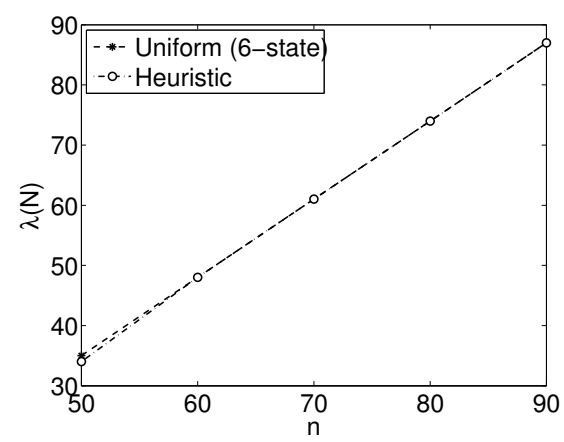

(f) capacity-Uniform (6-State)

Fig. 6. Comparison of the proposed heuristic and the exact simulation results (a) - (c) for optimal relay 1 placement and (d) - (f) for the min-cut capacity.

Theorem V.1. If $\mathcal{N}$ is a $(k, r)$ line network, then

$$
\lambda(\mathcal{N})=\lambda_{l}(\mathcal{N}) .
$$

Proof: Let $C_{i}=\left\{u_{0}, u_{1}, u_{2}, \cdots, u_{i}\right\}$ for $i \in$ $\{1,2, \cdots, k\}$. Let $\mathcal{T}_{C_{i}, \bar{C}_{i}}$ denote the transfer matrix corresponding to the cut $C_{i}$. As before, we denote by $\left|\mathcal{T}_{C_{i}, \bar{C}_{i}}\right|$ the binary rank of $\mathcal{T}_{C_{i}, \bar{C}_{i}}$. Recall that $\lambda\left(C_{i}\right)=\left|\mathcal{T}_{C_{i}, \bar{C}_{i}}\right|$. We will first show that

$$
\begin{aligned}
\lambda\left(C_{i}\right) & =\left|\mathcal{T}_{u_{i}, \bar{C}_{i}}\right| \\
& =n-c r_{i, i+1}
\end{aligned}
$$

where, $r_{i, i+1}$ is the distance between $u_{i}$ and $u_{i+1}$. We establish (18) as follows: For each $j \in\{0,1, \cdots, i-1\}$, we have

$$
\mathcal{T}_{u_{j}, \bar{C}_{i}}=S^{c r_{j, i}} \mathcal{T}_{u_{i}, \bar{C}_{i}} .
$$

Thus, it follows that

$$
\mathcal{T}_{C_{i}, \bar{C}_{i}}=\left(\begin{array}{c}
\mathcal{T}_{u_{i}, \bar{C}_{i}} \\
S^{c r_{i-1, i}} \mathcal{T}_{u_{i}, \bar{C}_{i}} \\
\vdots \\
S^{c r_{0, i} i} \mathcal{T}_{u_{i}, \bar{C}_{i}}
\end{array}\right) .
$$

Now, (18) readily follows from (19). Thus, we have the following alternative characterization of $\lambda_{l}(\mathcal{N})$ :

$$
\lambda_{l}(\mathcal{N})=\min _{j \leq k} n-c r_{j, j+1} .
$$

Now let $C$ be an arbitrary cut. $C$ must contain a node $u_{l}$ such that $u_{l+1} \notin C$. Thus, $\mathcal{T}_{u_{l}, u_{l+1}}$ is a submatrix of $\mathcal{T}_{C, \bar{C}}$ and consequently, we have

$$
\begin{aligned}
\left|\mathcal{T}_{C, \bar{C}}\right| & \geq\left|\mathcal{T}_{u_{l}, u_{l+1}}\right| \\
& =n-c r_{l, l+1} \\
& \geq \min _{j \leq k} n-c r_{j, j+1} \\
& =\lambda_{l}(\mathcal{N}) .
\end{aligned}
$$

Since the cut $C$ is arbitrary, we have that $\lambda(\mathcal{N}) \geq \lambda_{l}(\mathcal{N})$. The reverse inequality is obvious and hence the proof is complete.

Theorem V.2. If $\mathcal{N}$ is a $(k, r)$ line network, then

$$
\lambda(\mathcal{N}) \leq n-c\left\lceil\frac{r}{k+1}\right\rceil
$$

and the upper bound is achievable by placing the relays uniformly.

Proof: From Theorem V.1 and (20), we have

$$
\begin{aligned}
\lambda(\mathcal{N}) & =\min _{j \leq k} n-c r_{j, j+1} \\
& =n-c \max _{j \leq k} r_{j, j+1} \\
& \leq n-c\left\lceil\frac{r}{k+1}\right\rceil .
\end{aligned}
$$

Furthermore, the relays can be placed uniformly such that the distance between any two nodes is at most $\left\lceil\frac{r}{k+1}\right]$. Thus, the upper bound is achievable and the claims are established. 


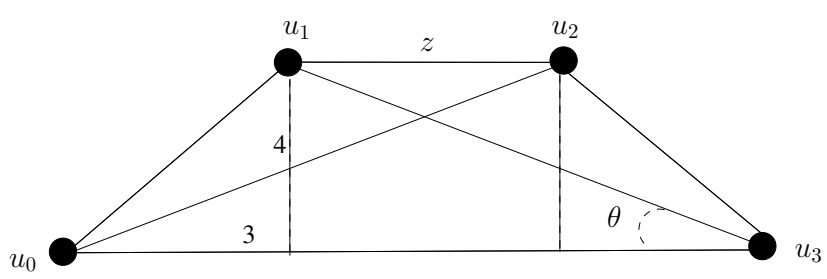

Fig. 7. Network $\mathcal{N}_{z}$ used to illustrate that uniform placement may be arbitrarily worse compared to optimal relay placement.

\section{B. Non-uniform relay placement on the plane}

Now we discuss an example of a two-relay network where, by placing relays in $2-D$ grid, we avoid the signal alignment and as a result obtain a large capacity advantage as compared to the two-relay line network without fading. Consider the $(2,18)$ network $\mathcal{N}_{z}$ shown in Figure 7 parameterized by the distance between the relays $u_{1}$ and $u_{2}$. For this network, we have

$$
\begin{aligned}
\theta & =\tan ^{-1}\left(\frac{4}{z+3}\right) \\
r_{0,2} & =\frac{z+3}{\cos (\theta)} \\
r_{1,3} & =\frac{z+3}{\cos (\theta)} \\
r_{0,3} & =z+6 .
\end{aligned}
$$

Now for $C_{4}=\left\{u_{0}, u_{1}\right\}$ and $n \geq z-6$, we have

$$
\mathcal{T}_{C_{4}, \bar{C}_{4}}=\left(\begin{array}{cc}
{\left[\begin{array}{cc}
0 & I_{n-c z} \\
0 & 0
\end{array}\right]} & {\left[\begin{array}{cc}
0 & I_{\left\lceil n-c r_{1,3}\right\rceil} \\
0 & 0
\end{array}\right]} \\
{\left[\begin{array}{cc}
0 & I_{\left\lceil n-c r_{0,2}\right\rceil} \\
0 & 0
\end{array}\right]} & \left.\left[\begin{array}{cc}
0 & I_{n-c(z+6)} \\
0 & 0
\end{array}\right]\right)
\end{array}\right.
$$

For all large $z$ and $n \geq z-6$, we have

$$
\left\lceil n-c r_{0,2}\right\rceil=\left\lceil n-c r_{1,3}\right\rceil=n-c(z+3) .
$$

Consequently, for all large $z$, we also have

$$
\mathcal{T}_{C_{4}, \bar{C}_{4}}=\left(\begin{array}{cc}
{\left[\begin{array}{cc}
0 & I_{n-c z} \\
0 & 0
\end{array}\right]} & {\left[\begin{array}{cc}
0 & I_{n-c(z+3)} \\
0 & 0
\end{array}\right]} \\
{\left[\begin{array}{cc}
0 & I_{n-c(z+3)} \\
0 & 0
\end{array}\right]} & \left.\left[\begin{array}{cc}
0 & I_{n-c(z+6)} \\
0 & 0
\end{array}\right]\right)
\end{array}\right.
$$

It is easy to verify that

$$
\begin{aligned}
\left|\mathcal{T}_{C_{4}, \bar{C}_{4}}\right| & =n-c z+n-c z-c 6 \\
& =2 n-2 c z-c 6 .
\end{aligned}
$$

Similarly for $C_{1}=\left\{u_{0}\right\}$ or $C_{2}=\left\{u_{0}, u_{1}, u_{2}\right\}$, we have

$$
\left|\mathcal{T}_{C_{1}, \bar{C}_{1}}\right|=\left|\mathcal{T}_{C_{2}, \bar{C}_{2}}\right|=n-c 5 \text {. }
$$

It follows that

$$
\lambda\left(\mathcal{N}_{z}\right)=\min \{2 n-2 c z-c 6, n-c 5\} .
$$

Let $\mathcal{N}$ be any $(2, z+6)$ line network. From Theorem V.2, we have

$$
\lambda(\mathcal{N}) \leq\lceil n-c(z+6) / 3\rceil .
$$

Comparing (21) and (22), for all large $z$ and for all $n \geq$ $2 c z+1$, we have

$$
\begin{aligned}
\lambda\left(\mathcal{N}_{z}\right) & \geq n-5 c \\
\lambda\left(\mathcal{N}_{z}\right)-\lambda(\mathcal{N}) & \geq n-5 c-(\lceil n-c(z+6) / 3\rceil) \\
& \geq n-5 c-(n-c z / 3-2 c+1) \\
& \geq c z / 3-3 c-1 .
\end{aligned}
$$

From the last inequality, it is clear that as $z$ grows to $\infty$ (and if $n$ grows as $2 c z+1$ ), placing the relays uniformly between the source and the receiver is arbitrarily worse compared to the trapezoidal arrangement in $\mathcal{N}_{z}$. This fact along with the result that the deterministic network approximates the corresponding Gaussian relay network can be used to show that there is an unbounded gain to placing the relays in a trapezoidal arrangement as in $\mathcal{N}_{z}$ compared to placing them on a line in Gaussian networks.

\section{CONCLUSIONS}

In this paper we were interested in the placement of relays on a line grid that maximizes the min-cut capacity of the corresponding deterministic network. We observed that for low power, a uniform placement is optimal, while as the power the nodes utilize increases, the relays tend to approach the sink and the destination respectively. We also proposed a heuristic for the min-cut value calculation, that we investigated through simulation results.

\section{REFERENCES}

[1] S. Avestimehr, S N. Diggavi and D N C. Tse, "Wireless network information flow", Proceedings of Allerton Conference on Communication, Control, and Computing, Illinois, September 2007.

[2] S. Avestimehr, S N. Diggavi and D N C. Tse, "Approximate capacity of gaussian relay networks", IEEE Symposium on Information Theory (ISIT), Toronto, July 2008.

[3] S. Avestimehr, S N. Diggavi and D N C. Tse, "Wireless network information flow: A Deterministic Approach", http://arxiv.org/abs/0906.5394, 2009.

[4] J. Ebrahimi and C. Fragouli, "Combinatorial algorithms for wireless information flow", arXiv:0909.4808, under submission.

[5] G. Kramer, I. Maric, and R.D. Yates, "Cooperative Communications", Foundations and Trends in Networking, vol. 1, no. 3-4, pp. 271-425, 2006.

[6] J. N. Laneman, D. N. C. Tse, and G. W. Wornell, "Cooperative Diversity in Wireless Networks: Efficient Protocols and Outage Behavior", IEEE Trans. Inform. Theory, vol. 50, no. 12, pp. 3062-3080, Dec. 2004.

[7] M. Franceschetti, J. Bruck, and L. J. Schulman, "A random walk model of wave propagation", IEEE Trans. on Antennas and Propagation, pp. 1304-1317, May 2004.

[8] J. Cannons,. L. Milstein, and K. Zeger, "An Algorithm for Wireless Relay Placement", IEEE Transactions on Wireless Communications vol. 8, pp. 5564-5574, November 2009.

\section{APPENDIX A}

Proof of Theorem III.3. Let $\mathcal{N}_{\infty}$ denote the grid network in which the vertex $(0,0)$ is occupied by the source $u_{0,0}$, vertex $(0, r)$ by the receiver $u_{0, r}$, and every other vertex is occupied 
by a relay node (i.e., there are infinitely many relay nodes). It is clear that

$$
\lambda(\mathcal{N}) \leq \lambda\left(\mathcal{N}_{\infty}\right)
$$

Now let $C$ denote the cut $\left\{u_{0,0}\right\}$. We have

$$
\begin{aligned}
\lambda(C) & =\left(1-\frac{1}{2^{4}}\right)(n-c)+\frac{1}{2^{4}}(n-c-d) \\
& =n-c-d / 16 .
\end{aligned}
$$

Thus, we have the upper bound

$$
\begin{aligned}
\lambda(\mathcal{N}) & \leq n-c-d / 16 \\
& {\left[\text { from }(23),(24), \text { and } \lambda\left(\mathcal{N}_{\infty}\right) \leq \lambda(C)\right] }
\end{aligned}
$$

Now let $N_{2, r}$ denote a $(2, r)$ relay nodes in which the relays are optimally placed. For $k \geq 2$, we have

$$
\begin{aligned}
\lambda(\mathcal{N}) & \geq \lambda\left(\mathcal{N}_{2, r}\right) \\
& \geq n-c-d / 2 . \quad \text { [from Lemma III.2] }
\end{aligned}
$$

Finally, if $k=1$, it is obvious that the relay must be placed at equal distance from the source and the receiver and consequently, it is immediate that $\lambda(\mathcal{N})=n-c\left\lceil\frac{r}{2}\right\rceil-d / 2$.

\section{APPENDIX B}

Proof of Theorem III.4. For $C_{1}=\left\{u_{0}\right\}$, we have

$$
\begin{aligned}
\mathbb{E} \lambda\left(C_{1}\right)= & \mathbb{E} \max \left\{n-c r_{1}-\log X_{u_{0}, u_{1}}, n-c\left(z+r_{1}\right)\right. \\
- & \left.\log X_{u_{0}, u_{2}}, n-c\left(z+r_{1}+r_{2}\right)-\log X_{u_{0}, u_{3}}\right\} \\
= & \frac{1}{2}\left(n-c r_{1}\right)+\frac{1}{4}\left(n-c\left(z+r_{1}\right)\right) \\
& +\frac{1}{8}\left(n-c\left(z+r_{1}+r_{2}\right)\right)+\frac{1}{8}\left(n-c r_{1}-d\right) \\
= & n-c r_{1}-\frac{1}{4} c z-\frac{1}{8} c\left(z+r_{2}\right)-\frac{1}{8} d \\
= & n-c r_{1}-\frac{1}{2} c z-\frac{1}{8} c\left(r_{1}+2 r_{2}\right) .
\end{aligned}
$$

Similarly, for $C_{2}=\left\{u_{0}, u_{1}, u_{2}\right\}$ we have

$$
\lambda\left(C_{2}\right)=n-c r_{2}-\frac{1}{2} c z-\frac{1}{8} c\left(r_{2}+2 r_{1}\right)
$$

For ease of notation, let $b_{i, j}=-\log X_{u_{i}, u_{j}}$ (i.e., $b_{i, j}$ is a $0 / d$-valued random variable). For $C_{3}=\left\{u_{0}, u_{2}\right\}$ it holds that

$$
\begin{aligned}
& \lambda\left(C_{3}\right)=\mathbb{E} \operatorname{rk}\left(\mathcal{T}_{C_{3}, \bar{C}_{3}}\right) \\
& =\mathbb{E} \mathrm{rk}\left(\begin{array}{cc}
{\left[\begin{array}{cc}
0 & I_{n-c z-b_{2,1}} \\
0 & 0
\end{array}\right]} & \left.\left[\begin{array}{cc}
0 & I_{n-c r_{2}-b_{2,3}} \\
0 & 0
\end{array}\right]\right) \\
{\left[\begin{array}{cc}
0 & I_{n-c r_{1}-b_{0,1}} \\
0 & 0
\end{array}\right]} & \left.\left[\begin{array}{cc}
0 & I_{n-c r-b_{0,3}} \\
0 & 0
\end{array}\right]\right)
\end{array}\right. \\
& \geq \frac{1}{2}\left(n-c r_{1}\right)+\frac{1}{4}\left(n-c\left(z+r_{1}\right)\right) \\
& +\frac{1}{8}\left(n-c\left(z+r_{1}+r_{2}\right)\right)+\frac{1}{8}\left(n-c r_{1}-d\right) \\
& =\lambda\left(C_{1}\right) \text {. }
\end{aligned}
$$

Finally, consider $C_{4}=\left\{u_{0}, u_{1}\right\}$. For all $n>2 c r$, we have

$$
\begin{aligned}
& \lambda\left(C_{4}\right)= \mathbb{E} \operatorname{rk}\left(\mathcal{T}_{C_{4}, \bar{C}_{4}}\right) \\
&\left.\left.=\mathbb{E} \operatorname{rk}\left(\begin{array}{cc}
0 & I_{n-c z-b_{1,2}} \\
0 & 0
\end{array}\right] \quad\left[\begin{array}{cc}
0 & I_{n-c\left(z+r_{2}\right)-b_{1,3}} \\
0 & 0
\end{array}\right]\right) \quad\left[\begin{array}{cc}
0 & I_{n-c r-b_{0}, 3} \\
0 & 0
\end{array}\right]\right) \\
&= \frac{3}{16}(n-c z)+\frac{3}{16}(n-c z-d) \\
&+\frac{3}{16}\left(n-c z+n-c\left(z+r_{1}+r_{2}\right)\right) \\
&+\frac{2}{16}\left(n-c z+n-c\left(z+r_{1}+r_{2}\right)-d\right)+ \\
&+\frac{2}{16}\left(n-c z-d+n-c\left(z+r_{1}+r_{2}\right)\right) \\
&+\frac{3}{16}\left(n-c z-d+n-c\left(z+r_{1}+r_{2}\right)-d\right) \\
&=(n-c z-d / 2)+\frac{10}{16}\left(n-c\left(z+r_{1}+r_{2}\right)-d / 2\right) .
\end{aligned}
$$

Comparing (25)-(27) with (28), for large enough $n$, we have $\lambda\left(C_{4}\right) \geq \lambda\left(C_{i}\right)$ for $i=1,2,3$ and it follows that

$$
\begin{aligned}
\lambda\left(\mathcal{N}_{2}\right) & =\min \left\{\lambda\left(C_{1}\right), \lambda\left(C_{2}\right)\right\} \\
& \leq \frac{1}{2}\left(\lambda\left(C_{1}\right)+\lambda\left(C_{2}\right)\right) \\
& =n-\frac{1}{2} c r-\frac{3}{16} c\left(r_{1}+r_{2}\right) .
\end{aligned}
$$

The upper bound is achievable by choosing $r_{1}=r_{2}$. 